\title{
A comparison of calculated and reconstructed values of the HCS tilts with positions of bright coronal streamers during total solar eclipses in 1870-2001
}

\author{
Mykola I. Pishkalo ${ }^{1}$ \\ ${ }^{1}$ Astronomical Observatory of Kyiv National Taras Shevchenko University, \\ 3, Observatorna vul., Kyiv, 04053, Ukraine, email: pish@observ.univ.kiev.ua
}

\begin{abstract}
Angular positions of the brightest coronal streamers observed during the total solar eclipses (TSEs) in 1870-2001 are compared with calculated tilts of the heliospheric current sheet (HCS) in 1976-2001 and with reconstructed ones in 1870-1976.
\end{abstract}

\section{Introduction}

The shape and structure of the solar corona are known to change with the cyclicity of solar activity from asymmetric "minimum" corona with two large streamers directed along solar equator to almost symmetric "maximum" corona with many radial bright rays and streamers. As a rule, the brightest streamers are located over magnetic neutral lines in the photosphere or at the "source surface". Magnetic neutral line on the "source surface" reflects location of the HCS near the Sun. Here we compare angular positions of the brightest coronal streamers with calculated tilts of the HCS during TSEs in 1976-2001 and with reconstructed ones during TSEs in 1870-1976.

\section{Data and analysis}

Angular positions of streamers were found at the distances of 2.5-3.5 solar radii using known published structural drawings, isophotes and photographs, obtained for TSEs in 1870-2001.

Calculated tilts of the HCS were found as positions of points of intersection of magnetic neutral line and solar limb at the moment of the eclipse on the Hoeksema's synoptic charts of the solar corona (see, http://wso.stanford.edu).

Reconstructed values of the HCS tilts for the time interval before 1976 were found using relationship between calculated tilts and sunspot numbers in 1976-2003. We investigated correlation and cross-correlation between international relative sunspot numbers (http://sidc.oma.be) and the HCS tilts, calculated by Hoeksema in potential classic and radial approximations. Correlation coefficient for non-smoothed corresponding data is equal approximately 0.85 . Use of running averaging of the data allows to increase value of correlation coefficient to, for example, 0.96-0.98 after fourfold smoothing. We didn't find any time shift between the data sets; it confirms that reversals of the global solar magnetic fields occur at the maxima of solar activity. Correlation coefficients between mentioned parameters are shown in Table 1. To reconstruct the HCS tilts such linear equations were finally used: $t=14.9+0.364 \mathrm{~W}$ for classic model tilts and $t=1.0+0.379 \mathrm{~W}$ for radial model tilts. Here $t$ is the tilt's value in degrees, $W$ is sunspot (Wolf's) number. 


\begin{tabular}{|c|c|c|c|}
\hline & $\begin{array}{c}\text { Sunspot number } \\
\text { Cycles } 21-23 \\
\text { c. } 21 \text { c. } 22 \text { c. } 23\end{array}$ & $\begin{array}{c}\text { Classic tilt } \\
\text { Cycles 21-23 }\end{array}$ & $\begin{array}{c}\text { Radial tilt } \\
\text { Cycles } 21-23\end{array}$ \\
\hline Classic tilt & $\begin{array}{rl} & 0.92 \\
0.90 & 0.920 .85\end{array}$ & 1.00 & 0.97 \\
\hline Radial tilt & $\begin{array}{ll} & 0.90 \\
0.88 & 0.900 .78\end{array}$ & 0.97 & 1.00 \\
\hline
\end{tabular}

Table 1. Correlation coefficient for monthly smoothed sunspot numbers and the HCS tilts
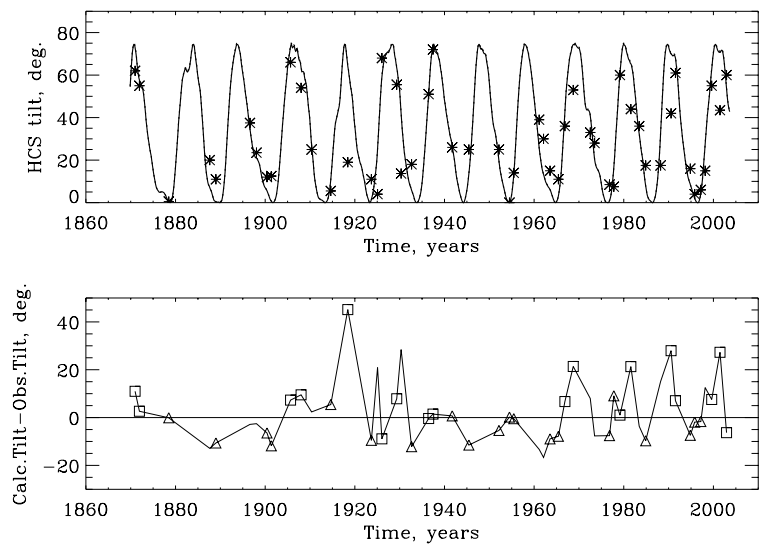

Figure 1. Comparison of reconstructed and observed tilts of the HCS in 1870-2001.

\section{Results}

The HCS tilts in 1976-2003 are in good correlation with sunspot numbers. This fact allows us to reconstruct HCS tilts before 1976 using known sunspot numbers. Figure 1 illustrates comparison of reconstructed tilts of the HCS with streamer positions observed during TSEs since 1870. Here the streamer angular positions are called by observed tilts of the HCS. Top panel shows reconstructed tilts normalized using maximal tilt values in cycles 21-23 by solid line and observed streamer positions by asterisks. Bottom panel shows differencies between calculated tilts and streamer positions. Mean differencies are -4.9 and $10.5 \mathrm{deg}$., r.m.s. differencies are 7.6 and $16.8 \mathrm{deg}$. for minima (triangles) and maxima (quadrates) periods of solar activity, respectively.

Observed positions of large coronal streamers during TSEs in 1976-2001 are in satisfactory agreement with calculated values of the HCS tilts, although sometimes their differencies reach 15-20 deg. As a whole, model values obtained using the classic potential model agree better with observed positions of coronal streamers than in case of the radial model.

\section{Acknowledgements}

Author thanks:

- T.Hoeksema and the Wilcox Observatory for coronal synoptic charts and the HCS data,

- P.Cugnon and the SIDC team for the sunspot number data, available via internet,

- the Organizing Committee for a possibility to participate in the Symposium. 